\title{
sartorius
}

\section{Fast, effective and safe adenovirus purification with Vivapure AdenoPACK kits}

\author{
The use of Sartorius membrane adsorbers incorporated in AdenoPACK units facilitates and speeds \\ up the purification step for adenovirus preparation, which can take days using traditional methods. \\ Ready-to-use kits are available for processing cell culture volumes from $20 \mathrm{ml}$ to $500 \mathrm{ml}$, permitting \\ preparation of up to $3 \times 10^{13}$ purified viral particles in only 2 hours. Plus, the method can be scaled up \\ for adenovirus purification in process scale.
}

Recombinant viral vectors are used to infect cells for expressing desired proteins for signal transduction experiments, gene-function analysis or gene-therapy research. Adenovirus is a nonenveloped dsDNA virus with a diameter of $80-90 \mathrm{~nm}$. Owing to its ability to infect most mammalian cell types (replicative and nonreplicative) and to replicate at high titers, it is one of the most widespread systems for viral gene transfer.

Various requirements need to be met when purifying adenovirus from cell culture for the above-mentioned applications. In the early phases of a research project, many different adenoviral constructs need to be screened. For this purpose, only small virus amounts are required. Gene-therapy experiments, however, require large amounts of virus. Additionally, the possibility of scaling up the method is a must when entering Phase I experiments ${ }^{1}$.

\section{Adenovirus purification with membrane chromatography}

Adenovirus is cultivated in HEK 293 cells and purified from the cell lysate as soon as cells start showing cytopathic effects. The most common purification method uses a cesium chloride $(\mathrm{CsCl})$ density gradient, in which the adenovirus is purified on the basis of its specific density. Density gradients, followed by the obligatory dialysis step to remove cytotoxic $\mathrm{CsCl}$, are very time-consuming and difficult to pursue with small and large cell-culture volumes. Because adenovirus is negatively charged under physiological conditions, it can effectively be purified using ion exchange chromatography ${ }^{2}$. Membrane chromatography offers a simple, scalable and fast alternative to the lengthy $\mathrm{CsCl}$ gradient purification method as well as purification with traditional chromatographic beads. Membrane adsorbers display a highly porous structure, with pores larger

\section{Noushin Delmdahl}

Sartorius AG, Weenderlandstr. 94-1008, D-37075 Goettingen, Germany. Correspondence should be addressed to N.D. (noushin.delmdahl@sartorius.com).

PUBLISHED ONLINE 21 J ULY 2006; DO:10.1038/ NMETH909 than 3,000 nm, and thus offer the adenoviral particles easy access to their charged surface ${ }^{3}$. Figure 1 shows an electron microscopic image of a membrane adsorber in comparison to common chromatography beads on the upper right side. The large membrane adsorber pores are easy to see, in contrast to the dense bead-surface structure.

Vivapure AdenoPACKs are ready-to-use membrane adsorber-based kits for convenient adenovirus purification at the laboratory scale. They contain all the filters, buffers, enzymes and other accessories needed for adenovirus purification. The AdenoPACK syringe filter arrangement incorporates the membrane adsorber for virus purification. The kits contain buffers, optimized for the purification of type 5 adenovirus, which is most frequently used for gene expression.

\section{Fast and simple type 5 adenovirus purification}

For a side-by-side comparison, we used Vivapure AdenoPACK100 for the purification of Ad-GFP (adenovirus expressing green fluorescent protein) from a 200-ml cell culture in comparison with the purification of the same virus from another 200-ml culture using the $\mathrm{CsCl}$ gradient purification method.

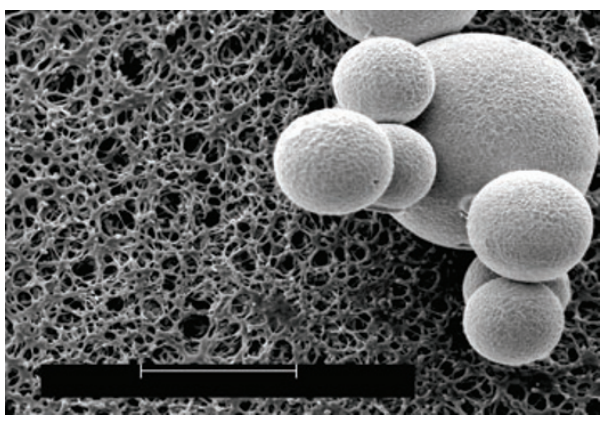

Figure 1 | Open porous structure of membrane adsorbers. An electron microscopic image comparing the open porous structure of the membrane adsorber to the much smaller chromatography bead pores. Scale bar, $50 \mu \mathrm{m}$. 
For both experiments, we infected low-passage HEK 293 cells with the adenovirus stock at a multiplicity of infection (m.o.i.) of 10-20 in 15 -cm plates containing $20 \mathrm{ml}$ of Dulbecco's modified Eagle medium (DMEM). We collected the cells when they started showing cytopathic effects. This step is not as critical when using the AdenoPACK kits, as the cell lysate and supernatant are both used for isolating the virus. When performing a $\mathrm{CsCl}$ gradient purification, the cells need to be collected before they release the virus into the supernatant, as only the cell pellet can be used.

For purification with AdenoPACK, we resuspended the harvested cell pellet in $10 \mathrm{ml}$ of DMEM and lysed it by three freeze-thaw cycles while storing $\sim 180 \mathrm{ml}$ of supernatant, which also contains adenoviruses, on ice. After lysis, we centrifuged the sample to remove cell debris and recombined it with the supernatant from the previous step. DNA digestion with supplied Benzonase ${ }^{\circledR}$ Nuclease was performed with the entire sample $(195 \mathrm{ml})$, followed by a final filtration, to prevent clogging of the AdenoPACK units. We prepared a second pellet obtained from 200-ml cell culture for a $\mathrm{CsCl}$ density gradient purification according to published protocols $(16 \mathrm{~h}$ at $100,000 \mathrm{~g}$, after which the viral band is recovered with a syringe, followed by a further $\mathrm{CsCl}$ density gradient, $16 \mathrm{~h}$ at 30,000g).

We equilibrated AdenoPACK units with loading buffer supplied in the kit and then loaded the previously prepared sample. The adenovirus particles are bound by the membrane adsorber's ionexchange ligands. Nonspecifically and loosely bound cell proteins are removed in a wash step before the final elution of the purified adenovirus. We quantified the virus particles by absorbance measurement at $260 \mathrm{~nm}$, taking one optical density unit at $260 \mathrm{~nm}$ to be equivalent to $10^{12}$ particles. The AdenoPACK kit purification yielded $3.5 \times 10^{12}$ viral particles, whereas the $\mathrm{CsCl}$ density gradient contained 1.5 viral particles.

We quantified the number of infectious particles using a commercial kit, resulting in the detection of approximately $5 \times 10^{10}$ infectious units from preparation made using both purification methods. The achieved virus purity is shown in a western blot (Fig. 2a). We then examined the viral preparation by a transfection assay for GFP expression in HeLa cells. Figure $\mathbf{2 b}$ shows a fluorescent microscopic image of HeLa cells transfected with Ad-GFP, after purification with the Vivapure AdenoPACK 100.

\section{Conclusions}

The AdenoPACK kit achieves purification results similar to those obtained via $\mathrm{CsCl}$ gradient purification in only 2 hours, a fraction of the time needed for a purification by $\mathrm{CsCl}$ gradient. A transfection assay with Ad-GFP in HeLa cells shows that adenovirus purified with the AdenoPACK kit is highly infective, and microscopic analysis of cell viability clearly indicates high purity and low levels of endotoxins in viral concentrates.

Membrane adsorbers are ideal for virus purification, as large flowthrough pores allow unrestricted access to the binding ligands and convective transport speeds up the purification process. Moreover, the method can be scaled up for adenovirus purification in process scale, a

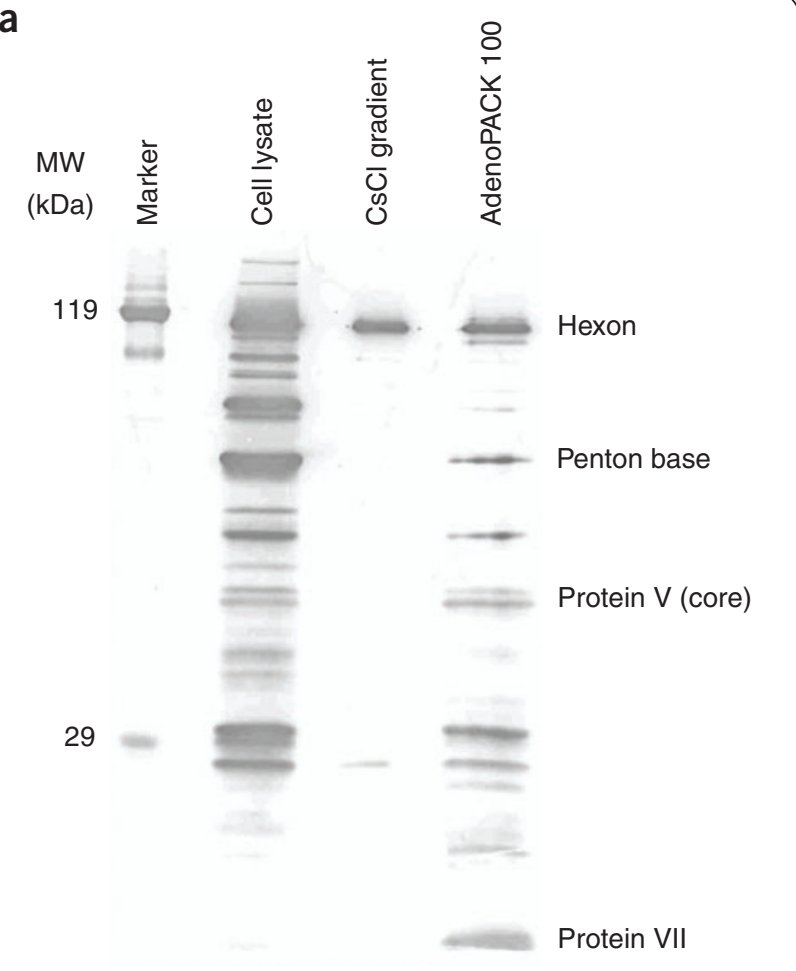

b

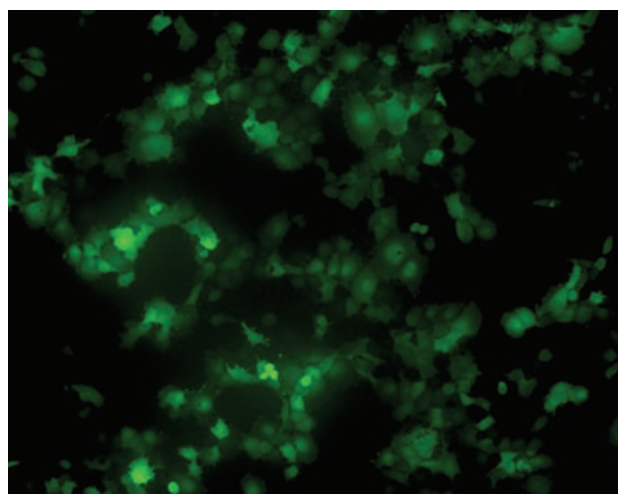

Figure $\mathbf{2}$ | Experimental purification results of Vivapure AdenoPACK. (a) A western blot comparing the effectiveness of adenovirus purification over two $\mathrm{CsCl}$ density gradients and using AdenoPACK 100. A rabbit polyclonal antibody was used for the detection of Ad5 hexon proteins. The second antibody was a goat-anti-rabbit-alkaline phosphatase conjugate. (b) Infection assay with GFPadenovirus and HeLa cells shows that the purified virus is highly infectious, as detected in the high number of fluorescent cells (kindly provided by G.L. Lux, University Hospital and University of Applied Sciences, Mannheim).

using the Sartorius SingleSep products, which are based on the same membrane adsorber technology.

1. Volpers, C. \& Kochanek, S. Adenoviral vectors for gene transfer and therapy. J. Gene Med. 6, S164-S171 (2004).

2. Delmdahl, N. Skalierbare membranchromatographie (Scalable membrane chromatography). Nachrichten aus der Chemie 54, 552-553 (2006).

3. Demmer, W. \& Nussbaumer, D. Large-scale membrane adsorbers. J. Chromatogr. A, 852, 73-81 (1999).

This article was submitted to Nature Methods by a commercial organization and has not been peer reviewed. Nature Methods takes no responsibility for the accuracy or otherwise of the information provided. 